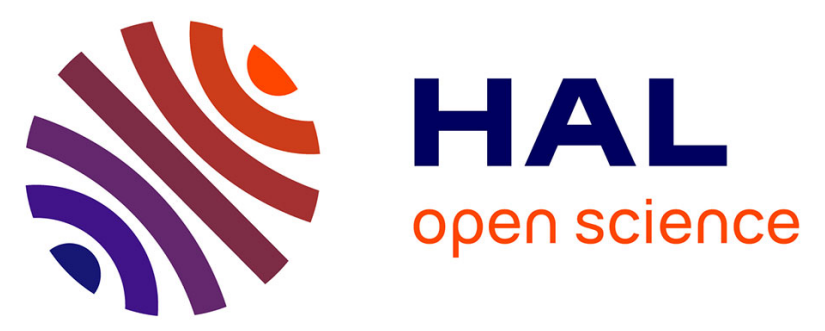

\title{
From canonical Hamiltonian to Port-Hamiltonian modeling application to magnetic shape memory alloys actuators.
}

Nandish Calchand, Arnaud Hubert, Yann Le Gorrec, Bernhard Maschke

\section{- To cite this version:}

Nandish Calchand, Arnaud Hubert, Yann Le Gorrec, Bernhard Maschke. From canonical Hamiltonian to Port-Hamiltonian modeling application to magnetic shape memory alloys actuators.. 4th Annual Dynamic Systems and Control Conference, DSCC'11., Oct 2011, Arlington, VA, United States. pp.1-8. hal-00720258

\section{HAL Id: hal-00720258 \\ https://hal.science/hal-00720258}

Submitted on 24 Jul 2012

HAL is a multi-disciplinary open access archive for the deposit and dissemination of scientific research documents, whether they are published or not. The documents may come from teaching and research institutions in France or abroad, or from public or private research centers.
L'archive ouverte pluridisciplinaire HAL, est destinée au dépôt et à la diffusion de documents scientifiques de niveau recherche, publiés ou non, émanant des établissements d'enseignement et de recherche français ou étrangers, des laboratoires publics ou privés. 


\section{FROM CANONICAL HAMILTONIAN TO PORT-HAMILTONIAN MODELING APPLICATION TO MAGNETIC SHAPE MEMORY ALLOYS ACTUATORS}

\author{
Nandish Calchand \\ Arnaud Hubert* \\ Yann Le Gorrec \\ FEMTO-ST Institute UMR 6174 \\ UFC/ENSMM/UTBM/CNRS, \\ 24 rue Alain Savary, 25000 Besançon \\ France \\ Email: nandish.calchand@femto-st.fr \\ Email: arnaud.hubert@femto-st.fr \\ Email: yann.gorrec@femto-st.fr
}

\author{
Bernhard Maschke \\ Laboratoire d'Automatique et de Génie des Procédés \\ Université Claude Bernard Lyon \\ 43 bd du 11 Novembre 1918 \\ 69622 Villeurbanne Cedex \\ France \\ Email: maschke@lagep.univ-lyon1.fr
}

\begin{abstract}
This paper presents the modelling of an actuator based on Magnetic Shape Memory Alloys (MSMA). The actuation principle relies on the ability of the material to change its shape under the application of a magnetic field. Previous models proposed by authors were based on canonical (symplectic) Hamiltonian modeling and thermodynamics of irreversible processes. These models, though physically cogent, are non-minimal differential algebraic dynamical models and hence less adapted for control purposes.This paper therefore proposes a modified and systemoriented modeling procedure which lends itself naturally to a port-Hamiltonian model. The latter is found to be a minimal realization of the above whereby interconnection between subsystems is clearly visible. Using Lagrange multipliers, constraints which arise due to causality and interconnection are expressed. In the last section, Differential Algebraic Equations (DAE) resulting from previous models are reduced to Ordinary Differential Equations (ODE) and by using coordinate transformations, constraints are decoupled from the system input/output. The resulting model is well-suited for control.
\end{abstract}

\section{INTRODUCTION}

In the context of miniaturization and performance improvement, actuators based on active materials are becoming very

\footnotetext{
*Address all correspondence to this author.
}

competitive because of compactness, high integration capability and precision. The range of applications and the area of new possibilities opened by these new devices are increasing rapidly $[1,2]$. Among the variety of active materials, piezoelectric materials are the most widely used as they have the desired properties needed for miniaturization, dynamics and control [3-6]. Nevertheless, some other materials are appearing based on electromagnetic actuation. Among them, the most promising is the Magnetic Shape Memory Alloys (MSMA) especially the $\mathrm{Ni}_{2} \mathrm{MnGa}$ alloy. Its crystallographic structure changes depending on the intensity of the magnetic field applied. These changes produce a strain which alters the material dimension. A model of such MSMA based mechatronic device can be found in [7]. It was based on canonical (symplectic) Hamiltonian modeling and thermodynamic of irreversible processes. These models though physically cogent and well-adapted for simulation purposes are non-minimal differential algebraic dynamical models and hence less adapted for control purposes. Based on this material, this paper will extend these works to suggest reduced order models that are better adapted for control. The new approach adopts a system-oriented point of view to manage multiphysic and mechatronic devices. This approach highlights the derivation of a port-Hamiltonian model of a MSMA based actuator from causal graph as it has been done in $[8,9]$ and [10]. The final objective is to obtain a minimum size model usable for control design using passivity techniques $[11,12]$. In the first 
part of the paper, the main characteristics of the MSMA are presented followed by some explanation about previous modeling works using "canonical" Hamiltonian method. Afterwards the advantages of an interconnection structure for multi-components system are presented. The interconnection and the associated port Hamiltonian model are obtained using linear graph theory and causality considerations. The system is divided into 2 subsystems, namely the "magnetic field generator" and the "energy converter (MSMA) + load". The two subsystems are then connected and the complete port-Hamiltonian model derived. A final step is devoted to model reduction. The objective is to transform the initial DAE system into a set of ODEs which could be used to implement new control strategies such as IDA-PBC for example.

\section{MSMA PROPERTIES AND CHARACTERISTICS Magnetic shape memory effect}

MSMA can be seen as a mixture of a classical shape memory alloy (SMA) and a magnetostrictive material. In short, the microscopic behaviour of MSMA is similar to the one of SMA $[13,14]$ but strain can not only be due to a martensite/austenite phase transformation but also due to a martensite reorientation under magnetic fields. In this second mode of working - martensite rearrangement -, MSMA can be assimilated to magnetostrictive materials (Terfenol-D) [15] except that it presents a much larger magneto-mechanical coupling ( $6 \%$ of maximal strain for $\mathrm{Ni}_{2} \mathrm{MnGa}$ instead of $0.16 \%$ for Terfenol-D). The magnetic actuation significantly increases the dynamical bandwidth of the crystallographic changes because it uses a magneto-mechanical energy conversion process instead of a thermo-mechanical process for classical SMA actuation. Since the first results fifteen years ago, MSMA materials have known some important improvements, namely the working temperature range and the maximum available strain. When actuated by magnetic fields, these materials now allow a large strain (up to $6 \%$ ) with a responsetime in the range of milliseconds as compared to tenth of seconds or even seconds for SMA (see $[16,17]$ for reviews). Currently, the most used MSMA are non-stoichiometric $\mathrm{Ni}_{2} \mathrm{MnGa}$ monocrystals but a lot of studies are also being conducted on thin films deposition and polycrystal samples $[18,19]$. Nevertheless, these latter types are less adequate for actuation applications because of a lower magneto-mechanical coupling. In this paper, only $\mathrm{Ni}_{2} \mathrm{MnGa}$ monocrystal is considered.

In this alloy, the martensite phase can appear in three different martensitic variants corresponding to the three possible crystallographic directions in the sample (see Fig. 1 (a)).

At high temperature, the MSMA sample is in austenitic phase (A) but after a cooling process, the austenite phase is transformed into a martensite phase without any favoured variants (M1, M2 and M3). If a mechanical stress is applied in a specific direction, then the fraction of variant with its short axis in this direction grows. If this stress is high enough then the sample will only contain this variant (for example M2 in Fig. 1 (b)). If

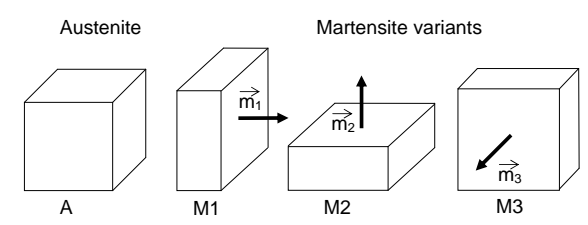

(a)

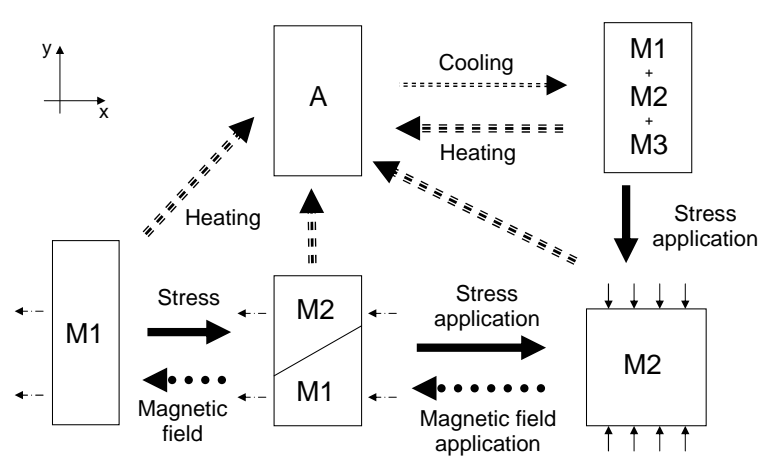

(b)

Figure 1. MSMA BEHAVIOR: (a) AUSTENITE PHASE AND THE THREE MARTENSITE VARIANTS, (b) MARTENSITIC REORIENTATION: EFFECTS OF MECHANICAL STRESS, MAGNETIC FIELD AND TEMPERATURE.

the stress decreases, the volume fraction of the M2 variant will also decrease but with a large thermo-magneto-mechanical hysteresis. In a similar way, if a magnetic field is applied, the variant with its easy magnetization direction in the field direction, is favoured. For $\mathrm{Ni}_{2} \mathrm{MnGa}$ MSMA, the easy magnetization direction is the same as the short axis of the martensite variant. In Fig. 1 (b), if magnetic and stress fields are orthogonal, they both favour a different variant of martensite (M1 or M2). The distribution between the magnetic field and the mechanical stress allows then to control the macroscopic strain. With a mechanical pre-stress, it is also possible to design an actuator driven by the magnetic field only. It should be stressed that by heating, austenite phase is recovered. More details about the structural properties of MSMA can be found in [16].

\section{Hamiltonian modeling of MSMA based actuators}

The MSMA based actuator considered in this paper is a simple device described in [7]. As depicted in Fig. 2, it is constituted by four components: (i) a control/supply electronic device (control board + PWM power supply, not depicted on the Fig. 2), (ii) a magnetic field generation device (coil + core), (iii) a MSMA sample and (iv) a mechanical load.

This mechatronic device was modeled in previous works using "canonical" Hamiltonian modeling and thermodynamics of irreversible processes $[7,20]$. This system is thermodynamically open because it posses a time-dependent external generalized 


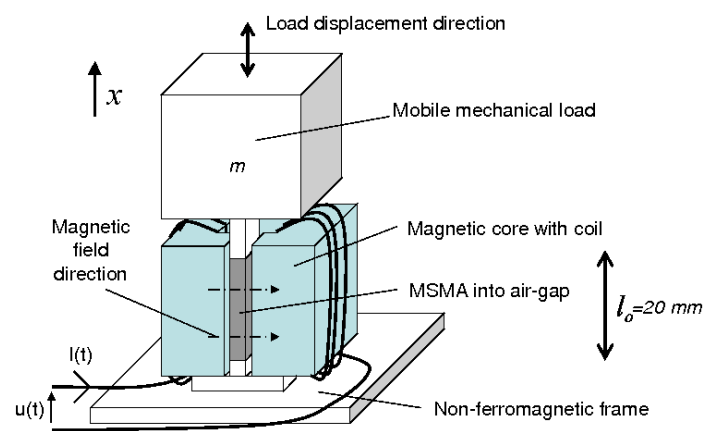

Figure 2. DESCRIPTION OF THE SIMPLE ACTUATOR USING MSMA.

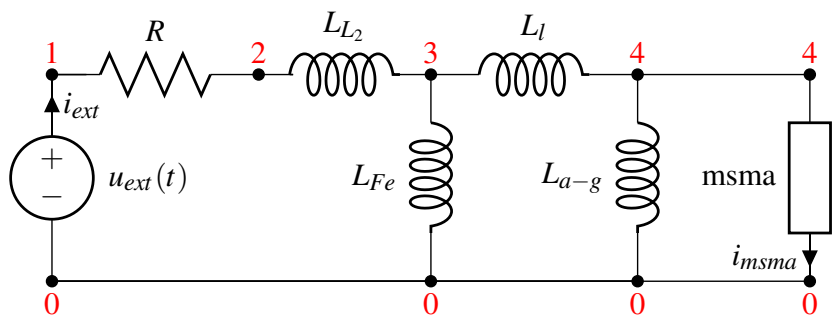

Figure 3. ELECTRICAL LUMPED PARAMETERS MODEL OF THE COMPONENTS i, ii AND iii OF THE MSMA ACTUATOR. $R$ IS THE ELECTRICAL RESISTANCE OF THE COIL. COIL INDUCTANCE AND MAGNETIC PATH ARE SEPARATED INTO EXTERNAL $\left(L_{2}\right)$, CORE $\left(L_{F e}\right)$, LEAKAGE $\left(L_{l}\right)$, AIR-GAP $\left(L_{a-g}\right)$ AND MSMA CONTRIBUTIONS.

force $f_{\text {ext }}(t)=u_{\text {ext }}(t)$ : the component $\mathrm{i}$ is considered as an external voltage source $u_{\text {ext }}(t)$. The component ii, coil + core, is modeled using an electrical lumped parameters model (see Fig. 3). This component contains conservative (inductance) and dissipative (resistance) parts. The component iii, MSMA, is modeled using thermodynamics of irreversible processes (Helmholtz free energy and dissipativity function) and contains conservative (elastic and magnetic), energy conversion (magneto-mechanic) and dissipative (thermodynamic irreversibility) parts. The component iv contains finally conservative (kinetic) and dissipative (viscous friction) parts.

The interconnection between these components was taken into account using Lagrange multipliers technique applied to the 3 interconnection constraints (the supply current $i_{\text {ext }}$ must flow into the coil, the magnetic field into MSMA is generated by the coil and the MSMA strain $\varepsilon$ drives the load displacement). A conservative Hamiltonian function $\mathcal{H}$ and an extented Hamiltonian function $\mathcal{H}^{\prime}$ depending on the time $t, n$ generalized coordinates $\mathbf{q}$ and $n$ generalized momenta $\mathbf{p}$ was used in the modeling procedure:

The external generalized forces $\mathbf{f}_{\text {ext }}(\mathbf{q}, t)$ was taken into account in the variation of $\mathcal{H}^{\prime}$ by adding the influence of their virtual works $\delta \mathcal{W}_{\text {ext }}=\mathbf{f}_{\text {ext }}(\mathbf{q}, t) \cdot \delta \mathbf{q}$.

Dissipations by static and viscous frictions was taken into account by adding their dissipated energies variations $\delta Q_{S}(\mathbf{q})$ and $\delta Q_{v}(\dot{\mathbf{q}})$. The dissipation by viscous friction $Q_{\mathcal{V}}(\dot{\mathbf{q}})$ was computed with a Rayleigh dissipation function $\mathcal{R}(\dot{\mathbf{q}})$ as $Q_{\mathcal{v}}(\dot{\mathbf{q}})=\int_{t_{1}}^{t_{2}} \mathcal{R}(\dot{\mathbf{q}}) d t$

The holonomic interconnection constraints $\mathbf{c}(\mathbf{q})=0$ was taken into account with a Lagrange multipliers technique by adding the term $\lambda \cdot \delta \mathbf{c}(\mathbf{q})$ to the variation $\delta \mathcal{H}^{\prime}$.

$$
\delta \mathcal{H}^{\prime}=\delta \mathcal{H}-\mathbf{f}_{\text {ext }} \cdot \delta \mathbf{q}-\delta Q_{s}-\delta Q_{v}+\lambda \cdot \delta \mathbf{c}
$$

As explained in [21], the dynamical equations can be computed using the Hamilton principle applied to the extented Hamiltonian function:

$$
\delta \int_{t_{1}}^{t_{2}} \mathbf{p} \dot{\mathbf{q}}-\mathcal{H}^{\prime} d t=0
$$

Using variational calculus on this expression, it results in the following $2 n$ Hamilton equations $(1 \leq k \leq n)$ :

$$
\left\{\begin{array}{l}
\dot{q}_{k}=\frac{\partial \mathcal{H}}{\partial p_{k}} \\
\dot{p}_{k}=-\frac{\partial \mathcal{H}}{\partial q_{k}}-\frac{\partial \mathcal{R}}{\partial \dot{q}_{k}}+\frac{\partial Q_{s}}{\partial q_{k}}+f_{\text {ext }, k}-\lambda_{k} \cdot \frac{\partial c_{k}}{\partial q_{k}}
\end{array}\right.
$$

As the device comprises 8 generalized coordinates, the Hamilton equations are a set of 16 equations, 8 associated with $\dot{\mathbf{q}}$ and 8 associated with $\dot{\mathbf{p}}$. Details about these equations and corresponding physical parameters can be found in [7]. These $2 n$ differential equations must be completed by $n_{c}=3$ algebraic constraint equations :

$$
c_{j}(\mathbf{q})=0 \quad 1 \leq j \leq n_{c}
$$

The model of this actuator using "canonical" Hamiltonian modeling is then a set of $2 n+n_{c}$ Differential Algebraic Equations. Simulations had been performed using the aforementioned model with load mass $m=1.44 \mathrm{~kg}$, length of MSMA sample $l_{0}=20 \mathrm{~mm}$, number of turns of coil $N=1500$, and resistance $R=61.8 \Omega$. A maximum strain of $\gamma=0.055$ due to martensite rearrangement can be obtained and this corresponds to an increase in length of the material of $x=1.1 \mathrm{~mm}$. Magnetic saturation of the material (easy magnetization axis) and core, obtained from experiments, were $0.65 \mathrm{~T}$ and $2.03 \mathrm{~T}$ respectively. Figure 4 shows the current and displacement for a step input of $60 \mathrm{~V}$ obtained by simulation and also the experimental measurements performed on the device at the Femto-ST Institute [7].

In the following section, it will be shown that if we adopt a system-oriented formalism as the port-Hamiltonian formalism, it will allow to drastically reduce the number of these modeling equations which are better suited for analysis, simulation and control. 

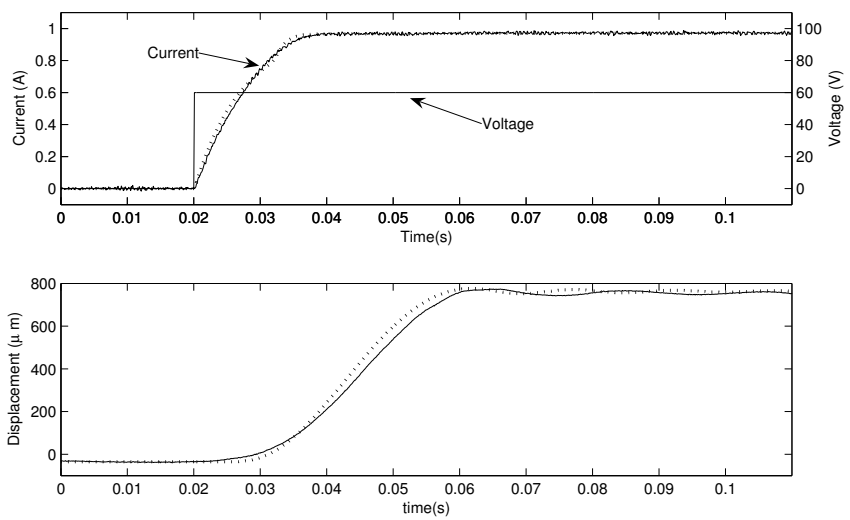

Figure 4. DYNAMIC BEHAVIOUR OF THE SYSTEM: VOLTAGE, CURRENT AND DISPLACEMENT VERSUS TIME (SIMULATION: DOTTED LINE, EXPERIMENTAL RESULTS: SOLID LINE).

\section{PORT-HAMILTONIAN MODELING}

The previous modelling procedure - "canonical" Hamiltonian modeling - gives $2 n+n_{c}$ DAE in the case of $n$ generalized coordinates constraint by $n_{c}$ interconnections. This model is physically cogent because it was experimentaly validated. It can adequately be used for simulation purposes using numerical tools. But, as noticed previously, as a non-minimal differential algebraic dynamical model, it is usually inadequate for control purpose. In the latter case, it is necessary to reduce them to gain insight into the design and control issues. This section will present how graph-oriented Port-Hamiltonian modeling allow to extend the "canonical" Hamiltonian formalism into a system-oriented modeling procedure well-suited for control issues [8-10].

The Port-Hamiltonian modeling [22-24] requires the systematic description of interconnections between each elementary component. For the lumped elements of the electrical network (see Fig. 3), this can be done using the linear graph theory $[8,25,26]$. For dipole/one port components, the linear graph theory proposes a systematic way to build the interconnection matrix $\mathcal{I}_{i o}$ linking external power-conjugated variables of each components according to their appearance in the tree $\mathcal{T}$ or the cotree $\overline{\mathcal{T}}$ of the graph. Depending on their causality, these external power-conjugated variables can be considered as output (o) or input (i) of this component. In this section, the complete actuator will be divided into two main subsystems: subsystem I corresponds to the grouping of previous component $\mathrm{i}$ and ii (electric and magnetic parts) ; subsystem II corresponds to the grouping of previous components iii and iv (thermodynamics and mechanics parts). The corresponding graph of subsystem I is depicted in the Fig. 5. In standard (sympletic) models of circuits (or Lagrangian models), states are written by first considering the (co-)energy variables of the (inductors) capacitors as independent. Then, the constraints between them induced by loops or cutsets are relaxed by introducing leakage currents or voltages (Lagrangian multi-

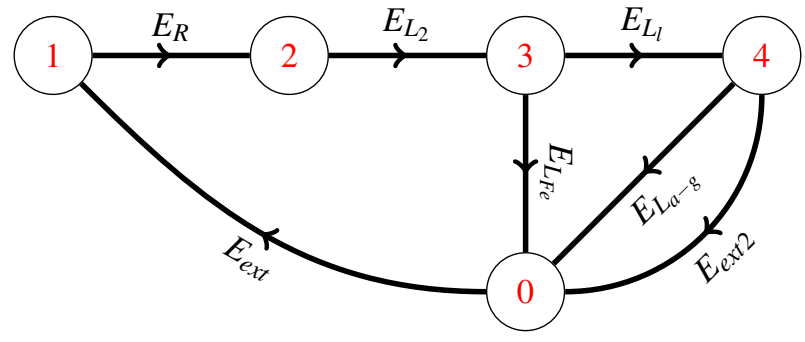

Figure 5. LINEAR GRAPH CORRESPONDING TO THE ELECTRICAL LUMPED-PARAMETERS MODEL OF THE COMPONENTS i AND ii (SEE FIG. 3: EXTERNAL VOLTAGE SUPPLY, COIL + CORE, EXTERNAL VOLTAGE 2: APPLIED TO MSMA). THE $E_{k}$ EDGE CORRESPONDS TO THE LUMPED PARAMETER $k$.

pliers). In our model, there is such kind of dependence because during the choice of a tree for the graph in the Fig. 5, the close inspection of prefered causality associated with each lumped elements shows that one state variable was not independent of others $\left(L_{2}, L_{l}, L_{F e}\right.$ forms a cutset). To formulate this dependence, a Lagrange multipliers technique associated with a leakage current $i_{\lambda 1}$ into a virtual additional branch/edge $E_{\lambda 1}$ was then adopted as depicted in Fig. 6. The constraint associated with this additional component/edge is:

$$
i_{\lambda 1}=i_{L_{2}}-i_{L_{F e}}-i_{L_{l}}=0
$$

Using the graph of Fig. 6, Eq. (6) gives the interconnection structure of subsystems I:

$$
\underbrace{\left(\begin{array}{c}
i_{\mathcal{T}} \\
u_{\overline{\mathcal{T}}}
\end{array}\right)}_{\mathbf{i}}=\underbrace{\left(\begin{array}{cc}
0 & -Q_{\overline{\mathcal{T}}} \\
-B_{\overline{\mathcal{T}}} & 0
\end{array}\right)}_{g_{i o}} \cdot \underbrace{\left(\begin{array}{c}
u_{\mathcal{T}} \\
i_{\overline{\mathcal{T}}}
\end{array}\right)}_{\mathbf{0}}
$$

where

$$
\begin{aligned}
& B_{\mathcal{T}}=-Q_{\overline{\mathcal{T}}}^{T}=\left(\begin{array}{cccc}
1 & 1 & 0 & 1 \\
0 & 0 & 0 & -1 \\
0 & 0 & 1 & -1 \\
0 & 0 & -1 & 0
\end{array}\right), \\
& u_{\mathcal{T}}=\left(\begin{array}{llll}
u_{R} & u_{\text {ext }} & u_{\text {ext } 2} & u_{\lambda 1}
\end{array}\right)^{T}, \\
& u_{\overline{\mathcal{T}}}=\left(\begin{array}{llll}
u_{L_{2}} & u_{L_{F e}} & u_{L_{l}} & u_{L_{a-g}}
\end{array}\right)^{T} \text {, } \\
& i_{\mathcal{T}}=\left(\begin{array}{llll}
i_{R} & i_{\text {ext }} & i_{\text {ext } 2} & i_{\lambda 1}
\end{array}\right)^{T} \text {, } \\
& i_{\overline{\mathcal{T}}}=\left(i_{L_{2}} i_{L_{F e}} i_{L_{l}} i_{L_{a-g}}\right)^{T} \text {. }
\end{aligned}
$$

In the Port-Hamiltonian formalism, a distinction between conservative $(\mathcal{H})$, dissipative $\left(Q_{s}+Q_{d}\right)$, external/source $\left(\mathcal{W}_{\text {ext }}\right)$ and Lagrange multipliers $(\boldsymbol{\lambda} \cdot \mathbf{c})$ terms is preserved: $\mathbf{i} \mapsto$ 


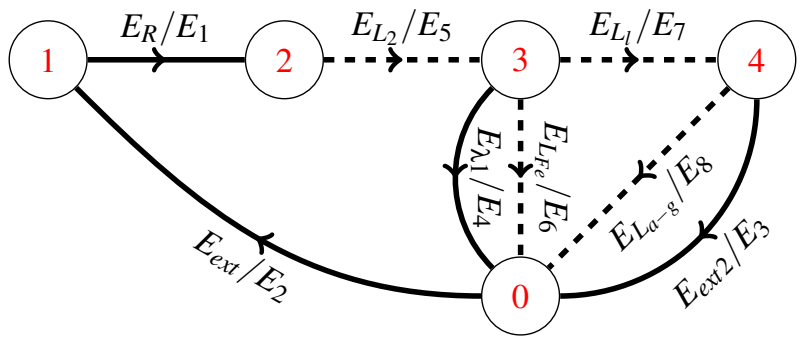

Figure 6. MODIFIED LINEAR GRAPH OF THE FIG. 5 WITH A LEAKAGE CURRENT $i_{\lambda 1}$ INTO A VIRTUAL ADDITIONAL BRANCH/EDGE $E_{\lambda 1}$ (TREE $\mathcal{T}$ : BOLD LINE; CO-TREE $\overline{\mathcal{T}}$ : DOTTED LINE) TO RESPECT THE PREFERED CAUSALITY FOR INDUCTANCES IN PARALLEL.

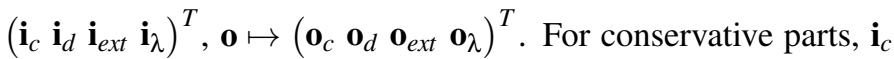
is the time rate of state variables $\mathbf{i}_{c}=\frac{d \mathbf{x}(t)}{d t}$ and $\mathbf{o}_{c}$ is the gradient of the conservative Hamiltonian function $\mathbf{o}_{c}=\frac{\partial \mathcal{H}}{\partial \mathbf{x}}$. In our example, it comes to the following equations:

\section{Subsystem I}

The conservative elements (inductances) are all grouped into the co-tree $\overline{\mathcal{T}}$ :

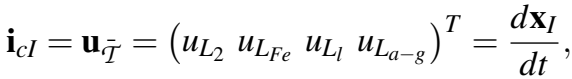

$$
\begin{aligned}
& \mathbf{o}_{c I}=\mathbf{i}_{\overline{\mathcal{T}}}=\left(i_{L_{2}} i_{L_{F e}} i_{L_{l}} i_{L_{a-g}}\right)^{T}=\frac{\partial \mathcal{H}_{I}}{\partial \mathbf{x}_{I}} .
\end{aligned}
$$

The state variables $\mathbf{x}_{I}$ are then defined as magnetic flux linkages in each inductance of the Fig. 3: $\mathbf{x}_{I}=\left(\phi_{L_{2}} \phi_{L_{F e}} \phi_{L_{l}} \phi_{L_{a-g}}\right)^{T}$.The tree $\mathcal{T}$ contains only dissipative, external or Lagrange multipliers terms:

$$
\begin{aligned}
& \mathbf{o}_{d I}=u_{R}, \\
& \mathbf{o}_{\text {ext } I}=\left(\begin{array}{ll}
u_{\text {ext }} & u_{\text {ext } 2}
\end{array}\right)^{T} \text {, } \\
& \mathbf{o}_{\lambda I}=u_{\lambda I} \text {. }
\end{aligned}
$$

As described in [24], when we consider a quadratic dissipative function, the corresponding Port-Hamiltonian equation of subsystem I for this device is:

$$
\frac{d \mathbf{x}_{I}}{d t}=\left(g_{I}-\mathbf{R}_{I}\right) \cdot \frac{\partial \mathcal{H}_{I}}{\partial \mathbf{x}_{I}}+\mathbf{G}_{I} \cdot\left(\begin{array}{c}
u_{\text {ext }} \\
u_{m s m a}
\end{array}\right)+\mathbf{A}_{I} \cdot u_{\lambda I}
$$

where in our case $g_{I}=\mathbf{0}$ and,

$$
\mathbf{R}_{I}=\left(\begin{array}{llll}
R & 0 & 0 & 0 \\
0 & 0 & 0 & 0 \\
0 & 0 & 0 & 0 \\
0 & 0 & 0 & 0
\end{array}\right), \mathbf{G}_{I}=\left(\begin{array}{cc}
-1 & 0 \\
0 & 0 \\
0 & -1 \\
0 & 1
\end{array}\right), \mathbf{A}_{I}=\left(\begin{array}{c}
-1 \\
1 \\
1 \\
0
\end{array}\right)
$$

\section{Subsystem II}

The subsystem II of this MSMA based actuator contains the parts iii and iv of the complete device (msma and load). The energetic behavior of MSMA will not be described in this paper and can be found in [20,27], its state is determined by 4 variables: the magnetic flux linkage $\phi_{m s m a}$ into MSMA, two thermodynamic internal variables $z$ and $p_{z}$ and its mechanical strain $\varepsilon$. The load is considered as a rigid body and contains only kinetic energy, function of the momentum $p_{\text {load }}$. In a similar way as subsystem I, we find for subsystem II:

$$
\begin{aligned}
& \mathbf{x}_{I I}=\left(\phi_{\text {msma }} z p_{z} \varepsilon p_{\text {load }}\right)^{T}, \\
& \mathbf{o}_{\text {cII }}=\frac{\partial \mathcal{H}_{I I}}{\partial \mathbf{x}_{I I}}=\left(i_{m s m a} \frac{\partial \mathcal{H}_{I I}}{\partial z} \frac{\partial \mathcal{H}_{I I}}{\partial p_{z}} \frac{\partial \mathcal{H}_{I I}}{\partial \varepsilon} \frac{\partial \mathcal{H}_{I I}}{\partial p_{\text {load }}}\right)^{T}, \\
& \mathbf{o}_{\text {extII }}=u_{\text {ext } 3} \quad(\text { voltage applied to msma, see Fig. 3). }
\end{aligned}
$$

The resulting Port-Hamiltonian equation is

$$
\frac{d \mathbf{x}_{I I}}{d t}=\underbrace{\left(\begin{array}{ccccc}
0 & 0 & 0 & 0 & 0 \\
0 & 0 & 1 & 0 & 0 \\
0 & -1 & \star & 0 & 0 \\
0 & 0 & 0 & 0 & 1 \\
0 & 0 & 0 & -1 & 0
\end{array}\right)}_{g_{I I}-\mathbf{R}_{I I}} \cdot \frac{\partial \mathcal{H}_{I I}}{\partial \mathbf{x}_{I I}}+\underbrace{\left(\begin{array}{l}
1 \\
0 \\
0 \\
0 \\
0
\end{array}\right)}_{\mathbf{G}_{I I}} \cdot u_{e x t 3}
$$

with $\star=\frac{\left(\partial \mathcal{P}_{\text {hyst }} / \partial \dot{z}\right)}{\dot{z}}$ where $\mathcal{P}_{\text {hyst }}$ is the dissipated power due to the irreversibility of msma (see [7]). It can be shown, from the $2^{\text {nd }}$ law of thermodynamics (Clausius-Duhem Inequality), that $\frac{\partial \mathcal{H}_{I I}{ }^{T}}{\partial \mathbf{x}_{I I}} \cdot \mathbf{R}_{I I} \cdot \frac{\partial \mathcal{H}_{I I}}{\partial \mathbf{x}_{I I}} \geq 0$ and hence subsystem II is passive.

\section{Interconnection between subsystems I and II}

These two subsystems are interconnected where subsystem II corresponds to $u_{\text {ext } 2}$ source for subsystem I and/or subsystem I correspond to $u_{\text {ext } 3}$ source for subsystem II. We therefore have:

$$
u_{L_{a-g}}=u_{\text {ext } 2}=u_{\text {ext } 3}=u_{m s m a}
$$

This interconnection produces a second causality problem, because MSMA and air-gap modeling present both an inductive behaviour and they have to be connected in parallel: one of the two corresponding states is dependent on the other. In a similar way as previously for subsystem I, this explicit dependence between state variables can be formulated using a Lagrange multiplier technique associated with a constraint defined by a second leakage current $i_{\lambda 2}$ parallel to the airgap and MSMA branch/edge:

$$
i_{\lambda 2}=-i_{L_{l}}+i_{L_{a-g}}+i_{m s m a}=0
$$


Finally the Port-Hamiltonian equation for interconnection of subsystems I and II is:

$$
\begin{aligned}
& \frac{d}{d t} \underbrace{\left(\begin{array}{c}
\mathbf{x}_{I} \\
\mathbf{x}_{I I}
\end{array}\right)}_{\mathbf{x}}=\underbrace{\left(\begin{array}{cc}
g_{I}-\mathbf{R}_{I} & 0 \\
0 & g_{I I}-\mathbf{R}_{I I}
\end{array}\right)}_{I_{-\mathbf{R}}} \cdot \underbrace{\left(\begin{array}{c}
\frac{\partial \mathcal{H}_{I}}{\partial \mathbf{x}_{I}} \\
\frac{\partial \mathcal{H}_{I I}}{\partial \mathbf{x}_{I I}}
\end{array}\right)}_{\frac{\partial \mathcal{H}}{\partial \mathbf{x}}} \\
& +\underbrace{\left(\begin{array}{c}
-1 \\
0 \\
0 \\
0 \\
0 \\
0 \\
0 \\
0 \\
0
\end{array}\right)}_{\mathbf{G}} \cdot \underbrace{u_{\text {ext }}}_{\mathbf{u}}+\underbrace{\left(\begin{array}{cc}
-1 & 0 \\
1 & 0 \\
1 & -1 \\
0 & 1 \\
0 & 1 \\
0 & 0 \\
0 & 0 \\
0 & 0 \\
0 & 0
\end{array}\right)}_{\mathbf{A}} \cdot \underbrace{\left(\begin{array}{l}
u_{\lambda 1} \\
u_{\lambda 2}
\end{array}\right)}_{\mathbf{u}_{\lambda}}
\end{aligned}
$$

With two constraint equations:

$$
\left\{\begin{array}{l}
i_{\lambda 1}=i_{L_{2}}-i_{L_{F e}}-i_{L_{l}}=0 \\
i_{\lambda 2}=-i_{L_{l}}+i_{L_{a-g}}+i_{m s m a}=0
\end{array}\right.
$$

These two constraint equations can be assigned in the PortHamiltonian formalism:

$$
\underbrace{\left(\begin{array}{l}
i_{\lambda 1} \\
i_{\lambda 2}
\end{array}\right)}_{\mathbf{y}_{\lambda}=\mathbf{i}_{\lambda}}=\underbrace{\left(\begin{array}{ccccccccc}
-1 & 1 & 1 & 0 & 0 & 0 & 0 & 0 & 0 \\
0 & 0 & -1 & 1 & 1 & 0 & 0 & 0 & 0
\end{array}\right)}_{\mathbf{A}^{T}} \cdot \underbrace{\left(\begin{array}{l}
\frac{\partial \mathcal{H}_{I}}{\partial \mathbf{x}_{I}} \\
\frac{\partial \mathcal{H}_{I I}}{\partial \mathbf{x}_{I I}}
\end{array}\right)}_{\frac{\partial \mathscr{H}}{\partial \mathbf{x}}}=0
$$

For quadratic dissipative systems, a Port-Hamiltonian output $\mathbf{y}$ power-conjugated with the external input $\mathbf{u}$ may be defined such as [28]:

$$
\frac{d \mathcal{H}}{d t}=-\frac{\partial \mathcal{H}^{T}}{\partial \mathbf{x}} \cdot \mathbf{R} \cdot \frac{\partial \mathcal{H}}{\partial \mathbf{x}}+\mathbf{y}^{T} \cdot \mathbf{u} \leq \mathbf{y}^{T} \cdot \mathbf{u}
$$

For the device considered in this paper, the computation gives:

$$
\begin{aligned}
\frac{d \mathcal{H}}{d t}= & \frac{\partial \mathcal{H}^{T}}{\partial \mathbf{x}} \cdot \frac{d \mathbf{x}}{d t} \\
= & \frac{\partial \mathcal{H}^{T}}{\partial \mathbf{x}} \cdot\left[(\mathcal{I}-\mathbf{R}) \cdot \frac{\partial \mathcal{H}}{\partial \mathbf{x}}+\mathbf{G} \cdot \mathbf{u}+\mathbf{A} \cdot \mathbf{u}_{\lambda}\right] \\
= & \frac{\partial \mathcal{H}^{T}}{\partial \mathbf{x}} \cdot \mathcal{J} \cdot \frac{\partial \mathcal{H}}{\partial \mathbf{x}}-\frac{\partial \mathcal{H}^{T}}{\partial \mathbf{x}} \cdot \mathbf{R} \cdot \frac{\partial \mathcal{H}}{\partial \mathbf{x}} \\
& +\left(\mathbf{G}^{T} \cdot \frac{\partial \mathcal{H}}{\partial \mathbf{x}}\right)^{T} \cdot \mathbf{u}+\left(\mathbf{A}^{T} \cdot \frac{\partial \mathcal{H}}{\partial \mathbf{x}}\right)^{T} \cdot \mathbf{u}_{\lambda}
\end{aligned}
$$

Because $\mathcal{J}=-\mathcal{I}^{T}$ (antisymmetric in accordance with Tellegen principle [29]) and $\mathbf{A}^{T} \cdot \frac{\partial \mathcal{H}}{\partial \mathbf{x}}=0$ (constraints), the first and the last parts of the second hands are nul and we obtain:

$$
\frac{d \mathcal{H}}{d t}=-\frac{\partial \mathcal{H}^{T}}{\partial \mathbf{x}} \cdot \mathbf{R} \cdot \frac{\partial \mathcal{H}}{\partial \mathbf{x}}+\left(\mathbf{G}^{T} \cdot \frac{\partial \mathcal{H}}{\partial \mathbf{x}}\right)^{T} \cdot \mathbf{u}
$$

The output $\mathbf{y}$ of this Port-Hamiltonian system is then defined as:

$$
\mathbf{y}=\mathbf{G}^{T} \cdot \frac{\partial \mathcal{H}}{\partial \mathbf{x}}=i_{L_{2}}
$$

The number of state variables is 9 in this Port-Hamiltonian modeling whereas it was 16 in the "canonical" Hamiltonian modeling. This system-oriented modeling procedure already allows to reduce the size of the dynamical problem by keeping only state variables instead of generalized coordinates and momenta. We also obtain a minimal realization of the system.

\section{MODEL REDUCTION}

The "canonical" Hamiltonian modeling procedure gives $2 n+n_{c}$ DAE in the case of $n$ generalized coordinates constraint by $n_{c}$ interconnections. The Port-Hamiltonian modeling procedure gives $n_{x}+n_{c x}$ DAE in the case of $n_{x}$ conservative components constraint by $n_{c x}$ equations. As previously noticed, it is still necessary to reduce them to gain insight into the design and control issues and especially to transform the DAE system into an ODE system. This section will present the reduction of DAE Port-Hamiltonian equations into a set of ODE Port-Hamiltonian equations by using changes of variables and state space projection according to [30]. The first step consists in decoupling the $n_{c x}$ Lagrange multipliers to $n_{x}-n_{c x}$ states of the system. It is done by the following change of coordinates: $\tilde{\mathbf{x}}=\mathbf{T}_{\mathbf{A}} \cdot \mathbf{x}$ with $\mathbf{T}_{\mathbf{A}}=(\mathbf{S} \mathbf{A})^{T}$ s.t. $\quad \mathbf{A}^{T} \cdot \mathbf{S}=0 . \quad \mathbf{S}$ being a real matrix of size $\left(n_{x}, n_{x}-n_{c x}\right)$. In our case the following matrix presents the required characteristics:

$$
\mathbf{S}^{T}=\left(\begin{array}{ccccccccc}
1 & 1 & 0 & 0 & 0 & 0 & 0 & 0 & 0 \\
0 & -1 & 1 & 1 & 0 & 0 & 0 & 0 & 0 \\
0 & 0 & 0 & 1 & -1 & 0 & 0 & 0 & 0 \\
0 & 0 & 0 & 0 & 0 & 1 & 0 & 0 & 0 \\
0 & 0 & 0 & 0 & 0 & 0 & 1 & 0 & 0 \\
0 & 0 & 0 & 0 & 0 & 0 & 0 & 1 & 0 \\
0 & 0 & 0 & 0 & 0 & 0 & 0 & 0 & 1
\end{array}\right)
$$

Because of the dissipative term $\mathbf{R}$ this first coordinate transformation is not sufficient. Indeed after this change of variables the Lagrange multipliers only act on the two last states of the system but these states remain connected to the other ones 
through $\mathbf{T}_{\mathbf{A}}(\mathcal{I}-\mathbf{R}) \mathbf{T}_{\mathbf{A}}^{T}$ due to the dissipative term $\mathbf{R}$. Furthermore the input is still coupled to the constraints as: $\mathbf{T}_{\mathbf{A}} \cdot \mathbf{G}=$

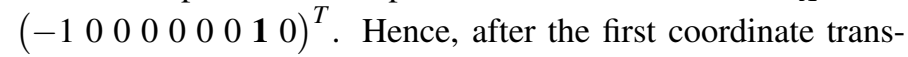
formation $\mathbf{T}_{\mathbf{A}}$ a second transformation $\mathbf{T}_{\mathbf{G}}$ is applied to remove this residual coupling coming from the dissipation term:

$$
\tilde{\tilde{\mathbf{x}}}=\mathbf{T}_{\mathbf{G}} \cdot \tilde{\mathbf{x}}=\underbrace{\mathbf{T}_{\mathbf{G}} \cdot \mathbf{T}_{\mathbf{A}}}_{\mathbf{T}} \cdot \mathbf{x}
$$

with the following matrix transformations:

$$
\mathbf{T}_{\mathbf{G}}=\left(\begin{array}{ccccccccc}
1 & 0 & 0 & 0 & 0 & 0 & 0 & 0 & 0 \\
0 & 1 & 0 & 0 & 0 & 0 & 0 & 0 & 0 \\
0 & 0 & 1 & 0 & 0 & 0 & 0 & 0 & 0 \\
0 & 0 & 0 & 1 & 0 & 0 & 0 & 0 & 0 \\
0 & 0 & 0 & 0 & 1 & 0 & 0 & 0 & 0 \\
0 & 0 & 0 & 0 & 0 & 1 & 0 & 0 & 0 \\
0 & 0 & 0 & 0 & 0 & 0 & 1 & 0 & 0 \\
1 & 0 & 0 & 0 & 0 & 0 & 0 & 1 & 0 \\
0 & 0 & 0 & 0 & 0 & 0 & 0 & 0 & 1
\end{array}\right), \mathbf{T}=\left(\begin{array}{ccccccccc}
1 & 1 & 0 & 0 & 0 & 0 & 0 & 0 & 0 \\
0 & -1 & 1 & 1 & 0 & 0 & 0 & 0 & 0 \\
0 & 0 & 0 & 1 & -1 & 0 & 0 & 0 & 0 \\
0 & 0 & 0 & 0 & 0 & 1 & 0 & 0 & 0 \\
0 & 0 & 0 & 0 & 0 & 0 & 1 & 0 & 0 \\
0 & 0 & 0 & 0 & 0 & 0 & 0 & 1 & 0 \\
0 & 0 & 0 & 0 & 0 & 0 & 0 & 0 & 1 \\
0 & 2 & 1 & 0 & 0 & 0 & 0 & 0 & 0 \\
0 & 0 & -1 & 1 & 1 & 0 & 0 & 0 & 0
\end{array}\right)
$$

The change of states $\mathbf{x} \mapsto \tilde{\tilde{\mathbf{x}}}$ gives the following Port-Hamiltonian equations:

$$
\left\{\begin{array}{l}
\frac{d \tilde{\tilde{\mathbf{x}}}}{d t}=\mathbf{T} \cdot(\mathcal{J}-\mathbf{R}) \cdot \mathbf{T}^{T} \cdot \frac{\partial \tilde{\tilde{\mathcal{H}}}}{\partial \tilde{\tilde{\mathbf{x}}}}+\mathbf{T} \cdot \mathbf{G} \cdot \mathbf{u}+\mathbf{T} \cdot \mathbf{A} \cdot \mathbf{u}_{\lambda} \\
\mathbf{y}=(\mathbf{T} \cdot \mathbf{G})^{T} \cdot \frac{\partial \tilde{\tilde{\mathcal{H}}}}{\partial \tilde{\tilde{\mathbf{x}}}} \\
\mathbf{y}_{\lambda}=(\mathbf{T} \cdot \mathbf{A})^{T} \cdot \frac{\partial \tilde{\mathcal{H}}}{\partial \tilde{\tilde{\mathbf{x}}}}=0
\end{array}\right.
$$

With the following state vectors and matrix:

$$
\tilde{\tilde{\mathbf{x}}}=\left(\begin{array}{c}
\phi_{L_{2}}+\phi_{L_{F e}} \\
-\phi_{L_{F e}}+\phi_{L_{l}}+\phi_{L_{a-g}} \\
\phi_{L_{a-g}}-\phi_{m s m a} \\
z \\
p_{z} \\
\varepsilon \\
p_{\text {load }} \\
2 \phi_{L_{F e}}+\phi_{L_{l}} \\
-\phi_{L_{l}}+\phi_{L_{a-g}}+\phi_{m s m a}
\end{array}\right), \mathbf{T} \cdot \mathbf{A}=\left(\begin{array}{cc}
0 & 0 \\
0 & 0 \\
0 & 0 \\
0 & 0 \\
0 & 0 \\
0 & 0 \\
0 & 0 \\
3 & -1 \\
-1 & 3
\end{array}\right)
$$

$$
\mathbf{T} \cdot(\mathcal{J}-\mathbf{R}) \cdot \mathbf{T}^{T}=\left(\begin{array}{ccccccccc}
-R & 0 & 0 & 0 & 0 & 0 & 0 & 0 & 0 \\
0 & 0 & 0 & 0 & 0 & 0 & 0 & 0 & 0 \\
0 & 0 & 0 & 0 & 0 & 0 & 0 & 0 & 0 \\
0 & 0 & 0 & 0 & 1 & 0 & 0 & 0 & 0 \\
0 & 0 & 0 & -1 & * & 0 & 0 & 0 & 0 \\
0 & 0 & 0 & 0 & 0 & 0 & 1 & 0 & 0 \\
0 & 0 & 0 & 0 & 0 & -1 & 0 & 0 & 0 \\
0 & 0 & 0 & 0 & 0 & 0 & 0 & 0 & 0 \\
0 & 0 & 0 & 0 & 0 & 0 & 0 & 0 & 0
\end{array}\right), \mathbf{T} \cdot \mathbf{G}=\left(\begin{array}{c}
-1 \\
0 \\
0 \\
0 \\
0 \\
0 \\
0 \\
0 \\
0
\end{array}\right)
$$

This model reduction procedure allows finally to isolate the two constraint equations (algebraic) from the rest of the other equations (ordinary differential) as it can be seen in the last two rows of $\mathbf{T} \cdot(\mathcal{I}-\mathbf{R}) \cdot \mathbf{T}^{T}, \mathbf{T} \cdot \mathbf{G}$ and $\mathbf{T} \cdot \mathbf{A}$ : the corresponding 7 ODE can be solved independently of the 2 AE. The final 7 order model usable for control can be derived by using projection.

This reduced model can now be used in further steps to design an efficient control law for this smart material based actuator. These further steps will be detailed in subsequent publications.

\section{CONCLUSIONS}

This paper presents the modeling of a smart material based actuator. This device uses the strain of a Magnetic Shape Memory Alloys sample activated by magnetic fields to generate controlable motions. In the first section, the working principle of this device is explained and, based on previous works of authors, a "canonical" Hamiltonian model is summarized. In the second section, limits of this modeling are explained and advantages of system-oriented modeling are stressed for design and control purposes. A Port-Hamiltonian modeling is then proposed for the device. This one is a minimal realization of the previous model and allows to express explicitly the interconnections between subsystems with a Lagrange multipliers technique. Finally, in the last section, the paper explains how DAE resulting from previous models can be reduced to an ODE Port-Hamiltonian models. This model reduction leads to a well-adapted model for control. They could be used to design efficient control law based on recent works on the control of Port-Hamiltonian systems such as IDA-PBC and similar strategies $[11,12]$.

\section{ACKNOWLEDGMENT}

Authors would like to thank Prof. Christian Lexcellent (Femto-ST Institute, Besançon France) and Dr Jean-Yves Gauthier (LGEF laboratory, INSA Lyon, France) for dicussions and collaborations concerning the modeling of SMA and MSMA. This work was supported by the BQR project "Approche Hamiltonienne à ports pour la commande des systèmes à paramètres distribués : application à la commande des micro-systèmes", University of Franche-Comté, Besançon, France. 


\section{REFERENCES}

[1] Janocha, H., ed., 1999. Adaptronics and Smart Structures. Basics, materials, Design, and Applications. SpringerVerlag.

[2] Pons, J. L., 2005. Emerging Actuator Technologies: A Micromechatronic Approach. John Wiley and Sons.

[3] Bashash, S., and Jalili, N., 2009. "Robust adaptative control of coupled parallel piezo-flexural nanopositioning stages". IEEE/ASME Transactions on Mechatronics, 14(1), February, pp. 11-20.

[4] Merry, R., de Kleijn, N., van de Molengraft, N., and Steinbuch, M., 2009. "Using a walking piezo actuator to drive and control a high-precision stage". IEEE/ASME Transactions on Mechatronics, 14(1), February, pp. 21-31.

[5] Polit, S., and Dong, N. J., 2010. "Development of a high-bandwidth xy nanopositioning stage for high-rate micro-/nanomanufacturing”. IEEE/ASME Transactions on Mechatronics, 99, June, pp. 1-10.

[6] Peng, J., and Chen, D. B., 2010. "Modeling of piezoelectric-driven stick-slip actuators". IEEE/ASME Transactions on Mechatronics, 99, March, pp. 1-6.

[7] Gauthier, J. Y., Hubert, A., Abadie, J., Chaillet, N., and Lexcellent, C., 2008. "Nonlinear Hamiltonian modelling of magnetic shape memory alloy based actuators". Sensors \& Actuators A, 141, pp. 536-547.

[8] Maschke, B., van der Schaft, A. J., and Breedveld, P., 1995. "An intrinsic Hamiltonian formulation of the dynamics of LC-circuits". IEEE Transactions on Circuits and Systems I: Fundamental Theory and Applications, 42, pp. 73-82.

[9] Maschke, B. M., van der Schaft, A. J., and Breedveld, P. C., 1992. "An intrinsic Hamiltonian formulation of network dynamics: non-standard poisson structures and gyrators". Journal of the Franklin Institute, 329(5), pp. 923 - 966.

[10] Donaire, A., and Junco, S., 2009. "Derivation of inputstate-output port-Hamiltonian systems from bond graphs". Simulation Modelling Practice and Theory, 17(1), pp. 137 -151 .

[11] Maschke, B., Ortega, R., and van der Schaft, A. J., 2002. "Energy based Lyapunov functions for forced Hamiltonian systems with dissipation". IEEE Trans.on Autom. Control, 45(8), pp. 1498-1502.

[12] Ortega, R., van der Schaft, A. J., Maschke, B., and Escobar, G., 2002. "Interconnection and damping assignment passivity-based control of port-controlled Hamiltonian systems". Automatica, 38, pp. 585-596.

[13] Bhattacharya, K., 2003. Microstructure of Martensite: Why It Forms and How It Gives Rise to the Shape-Memory Effect. Oxford University Press.

[14] Lagoudas, D. C., ed., 2008. Shape Memory Alloys: Modeling and Engineering Applications. Springer-Verlag.

[15] du Tremolet de Lacheisserie, E., 1993. Magnetostriction: Theory and Applications of Magnetoelasticity. CRC Press.

[16] Söderberg, O., Ge, Y., Sozinov, A., Hannula, S.-P., and Lindroos, V. K., 2005. "Recent breakthrough development of the magnetic shape memory effect in Ni-Mn-Ga alloys". IOP Smart Materials and Structures, 14, pp. 223-335.

[17] Pons, J., Cesari, E., Seguì, C., Masdeu, F., and Santamarta, R., 2008. "Ferromagnetic shape memory alloys: Alternatives to ni-mn-ga". Materials Science and Engineering A, 481-482, pp. 57-65.

[18] Kohl, M., Brugger, D., and Krevet, B., 2006. "Ferromagnetic shape memory actuator for large 2 d optical scanning". In 19th IEEE International Conference on Micro Electro Mechanical Systems, Istanbul, Turkey.

[19] Kohl, M., Brugger, D., Ohtsuka, M., and Krevet, B., 2007. "A ferromagnetic shape memory actuator designed for large 2d optical scanning". Sensors and Actuators A, 135, pp. 92-98.

[20] Gauthier, J. Y., Lexcellent, C., Hubert, A., Abadie, J., and Chaillet, N., 2007. "Modeling rearrangement process of martensite platelets in a magnetic shape memory alloy $\mathrm{Ni}_{2} \mathrm{MnGa}$ single crystal under magnetic field and (or) stress action". Journal of Intelligent Material Systems and Structures, 18(3), pp. 289-299.

[21] Lanczos, C., 1986. The Variational Principe of Mechanics, Fourth edition. Dovers Publications, Reprint of 1970 University of Toronto Press.

[22] van der Schaft, A. J., 2000. L2-Gain and Passivity Techniques in Nonlinear Control. Springer-Verlag.

[23] Astolfi, A., and van der Schaft, A. J., eds., 2004. Lagrangian and Hamiltonian Methods for Nonlinear Control. Special Issue of European Journal of Control, Volume 10, Number 5. Hermes.

[24] Brogliato, B., Lozano, R., Maschke, B., and Egeland, O., 2007. Dissipative Systems Analysis and Control. Theory and applications, $2^{\text {nde }}$ edition. Springer-Verlag.

[25] Shearer, J. L., Murphy, A. T., and Richardson, H. H., 1967. Introduction to system dynamics. Addisson-Wesley.

[26] Koenig, H. E., Tokad, Y., and Kesavan, H. K., 1967. Analysis of Discrete Physical Systems. MacGraw-Hill.

[27] Gauthier, J. Y., Lexcellent, C., Hubert, A., Abadie, J., and Chaillet, N., 2011. "Magneto-thermo-mechanical modeling of a magnetic shape memory alloy ni-mn-ga single crystal". Annals of Solid and Structural Mechanics (in press).

[28] van der Schaft, A. J., 2006. "Port-Hamiltonian systems: an introductory survey". In Proceedings of the International Congress of Mathematicians, Madrid, Spain, pp. 13391364.

[29] Tellegen, B. D. H., 1952. "A general network theorem with applications". Philips Research Reports, 7, pp. 259-269.

[30] van der Schaft, A., and Maschke, B., 1994. "On the Hamiltonian formulation of non-holonomic mechanical systems.". Reports on Mathematical Physics, 34(2), pp. 225-233. 